\title{
Variational approach to the calculation of the lowest Wannier exciton state in wide type-II single semiconductor quantum wells
}

\author{
G.V. Vertsimakha \\ Institute for Nuclear Research, \\ 47, prospect Nauky, 03680 Kyiv, Ukraine, \\ Phone: +380 (44) 525-46-10; e-mail: avertsim@kinr.kiev.ua
}

\begin{abstract}
The possibility to change the spatial character of the Wannier exciton ground state in a wide single type-II semiconductor quantum well has been studied variationally. A heterostructure with the central layer forming a potential well for holes and a barrier for electrons has been considered. A trial function taking into account the possibility to shift the most probable position of hole from the center of the structure towards interfaces for reducing the distance to electron has been proposed. The exciton transition energy and binding energy were calculated for the structure based on the $\mathrm{ZnO}$ one. It has been shown that the proposed trial functions can be used for wide quantum wells for which it describes an exciton state with the carriers localized near the interfaces at a distance of the order of the Bohr radius for bulk exciton.
\end{abstract}

Keywords: exciton, quantum well, binding energy, variational calculation.

Manuscript received 12.01.16; revised version received 20.04.16; accepted for publication 08.06.16; published online 06.07.16.

\section{Introduction}

Quantum heterostructures with the type-II band alignment has been investigated for a long time due to promising physical properties: a long recombination lifetime as compared to that of type-I systems, lower exciton binding energy, strong dependence of the emission energy and oscillator strength on the well width [1-3], suppression of the Auger recombination rate [4], enhanced Stark-effect $[5,6]$ and others. These features are caused, first of all, by the band alignment between materials of the structure layers, which determines the spatial distribution of excited charge carriers. In a type-I structure, both electrons and holes are localized in the same layer of the structure, forming the spatially direct exciton. In type-II structures, a step-like band edge profile leads to creation of an indirect exciton with electron and hole separated across the interface, that is to say, localized in different layers of the structure. A suitable choice of structure parameters (semiconductor material combinations, alloy compositions and layer widths) offers a possibility to tune the indirect exciton properties, which is important for various applications like mid-infrared quantum well lasers [7], solar cells [8], infrared photodetectors [9-11], nonlinear optics [12], etc. In these heterostructures, the long lifetime allows 
creating a high concentration of carriers and, hence, using these structures for the study of collective exciton phenomena $[13,14]$.

The starting point in both fundamental researches like investigations of collective phenomena and practical applications of nanocrystals has to be the study of single-exciton states determining optical properties and providing a basis for understanding more complex problems. In a type-II single quantum well with a potential barrier for electrons and a potential well for holes (such a system will be considered in this paper) the symmetry of the problem makes it natural to suggest that the most probable spatial position of the hole is in the center of the well (Fig. 1a). However, if the well is sufficiently wide, and Coulomb interaction between carriers is strong enough, it might be energetically favorable for the carriers to reduce the distance between each other: hole could move from the well center towards interfaces and electron also could approach closer to the interfaces in the external layers (Fig. 1b). In order to check availability of this state, one can calculate its energy using the variational approach with a trial function taking into account the possibility of this noncentral spatial position of hole. If the energy of the exciton state with the non-central position of the hole in the well turns out to be lower than the energy of the state with the central hole position, this state is plausible.

Variational approach is traditional for calculations of the Wannier exciton spectra in type-II single quantum wells and superlattices [15-18]. As a rule, a trial exciton function containing as multipliers one-particle wave functions of the electron and hole states in the quantum wells is used [16]. These functions are well known for type-I single quantum wells and for superlattices of both I and II types, but there are some difficulties in determining the one-particle part of the exciton trial function for a type-II single quantum well. The point is that only one particle in a type-II single quantum well, electron or hole, is confined in the well layer, whereas the second particle would be not localized in the absence of the Coulomb interaction. In $[17,18]$, the method of the exciton spectra calculation for these structures combining variational and numerical calculations was proposed, and it has been used in this paper. Within this approach, it is supposed that a carrier, confined in the quantum well, creates an effective Coulomb potential localizing the second carrier near the potential barrier.

In this paper, a trial function for variational calculation of the lowest Wannier exciton state energy in a type-II single quantum well has been proposed in order to examine the possibility to exist for the exciton state with the non-central most probable hole position in the potential well.

\section{Method of calculations}

In order to find the exciton energy by using variational calculations, one has to choose a proper trial function for the particular structure. We consider a type-II single quantum well depicted in Fig. 1, the central layer of which forms a quantum barrier for electron in the conduction band of the structure and a quantum well for hole in its valence band, so the Hamiltonian of the system can be written as

$$
H=H_{0}+V_{e}\left(z_{e}\right)+V_{h}\left(z_{h}\right),
$$

where $H_{0}$ is the Hamiltonian of free Wannier exciton, $\vec{r}_{e(h)}=\left(z_{e(h)}, \vec{\rho}_{e(h)}\right)-$ electron (hole) coordinate, $\vec{\rho}_{e(h)}-$ electron (hole) coordinate in the plane of the layers, the axis $z$ coincides with the direction of the structure growth, $V_{e}\left(z_{e}\right)$ and $V_{h}\left(z_{h}\right)$ are the potential profiles for electron and hole, respectively.

As a rule, for quantum wells the $1 \mathrm{~S}$ exciton state trial function can be chosen in a form of a product of one-particle wave functions of electron $f_{e}\left(z_{e}\right)$ and hole $f_{h, c}\left(z_{h}\right)$ and an exciton-like function $\Phi_{e x}(r)$ describing the relative carrier movement due to the Coulomb interaction, namely:

$\Psi_{c}\left(\vec{r}_{e}, \vec{r}_{h}\right)=N_{c} f_{e}\left(z_{e}\right) f_{h, c}\left(z_{h}\right) \Phi_{e x}(r)$,

where $N_{c}$ is the normalization constant, and the $f_{h, c}\left(z_{h}\right)$ - function of the lowest state inherent to hole in the rectangular potential well:

$$
f_{h, c}\left(z_{h}\right)= \begin{cases}e^{-\kappa\left(\left|z_{h}\right|-L / 2\right),} & \left|z_{h}\right| \geq L / 2 \\ \cos \left(k z_{n}\right), & \left|z_{h}\right| \leq L / 2\end{cases}
$$

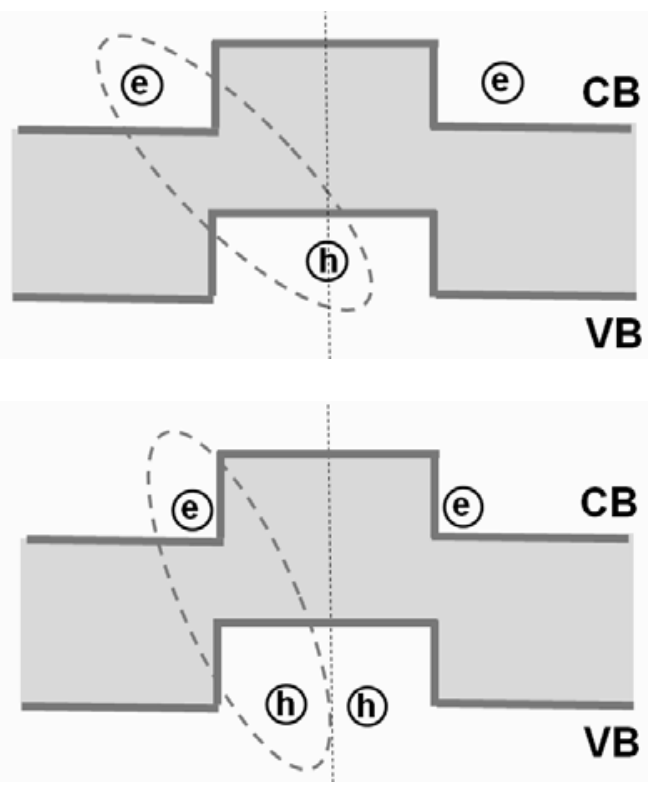

Fig. 1. Band structure of a type-II quantum well with a schematic picture of the exciton state with the (a) central and (b) non-central hole most probable positions. Hole and electron are depicted twice in order to emphasize that the state is symmetric in respect to the axis of symmetry of the structure. 
Here, the subscript "c" was chosen to emphasize that this function fixes the most probable central position of hole in the well. The parameters $k$ and $\kappa$, as usual, were calculated from the condition of function continuity. In order to describe a possible shift of hole towards interfaces, we will use the exciton function similar to (2), but with a different one-particle function for hole:

$$
\begin{aligned}
& \Psi_{n c}\left(\vec{r}_{e}, \vec{r}_{h}\right)=N_{n c} f_{e}\left(z_{e}\right) f_{h, n c}\left(z_{h}\right) \Phi_{e x}(r), \\
& f_{h, n c}\left(z_{h}, \gamma\right)= \begin{cases}e^{-\kappa\left(\left(\left|z_{h}\right|-L / 2\right)\right.}\left(e^{-\gamma z_{h}}+e^{\gamma z_{h}}\right), & \left|z_{h}\right| \geq L / 2 \\
\cos \left(k z_{n}\right)\left(e^{-\gamma z_{h}}+e^{\gamma z_{h}}\right), & \left|z_{h}\right| \leq L / 2\end{cases}
\end{aligned}
$$

where $\gamma$ is the variational parameter, which determines the shift of hole from the well center to its interfaces, $N_{n c}$ - normalization constant. If $\gamma=0$, the function (4) coincides with the usual function (2).

The trial function for the relative movement was chosen in the simplest form with a single variational parameter $\lambda$ :

$$
\Phi_{e x}(\rho)=\sqrt{\frac{1}{\pi \lambda^{3}}} \exp \left(-\frac{r}{\lambda}\right) .
$$

And, finally, in order to calculate variationally the exciton binding energy and the exciton transition energy, the numerical method proposed in $[17,18]$ was used. This method includes the numerical calculation of the one-particle function $f_{e}\left(z_{e}\right)$ for electron that is localized near the quantum well interface only due to the action of Coulomb attraction of hole. It should be noted that although in (2) and (4) the wave functions $f_{e}\left(z_{e}\right)$ and $\Phi_{e x}(\rho)$ have the same form and are designated alike, in fact their values are different for different trial functions because both the value of the variational parameter $\lambda$ and the electron function $f_{e}\left(z_{e}\right)$ are calculated for different one-particle hole functions $f_{h, c}\left(z_{h}\right)$ and $f_{h, n c}$.

For these calculations, the parameters corresponding to the $\mathrm{ZnO} / \mathrm{ZnO}_{1-\mathrm{x}} \mathrm{Te}_{\mathrm{x}} / \mathrm{ZnO}$ structures were chosen because of very strong Coulomb interaction. In this system, in some range of $\mathrm{Te}$ concentrations, the $\mathrm{ZnO}_{1-\mathrm{x}} \mathrm{Te}_{\mathrm{x}}$ layer forms a potential barrier for electrons and well for holes. The natural availability, stability, non-toxic nature, high luminous efficiency as well as mechanical and chemical robustness have made the wide gap $(3.3 \mathrm{eV})$ zinc oxide $(\mathrm{ZnO})$ and various $\mathrm{ZnO}$-based heterostuctures promising materials for optical devices and photovoltaic applications [8, 19, 20]. Recently, highly mismatched alloys like $\mathrm{ZnO}_{1-\mathrm{x}} \mathrm{Te}_{\mathrm{x}}, \mathrm{ZnO}_{1-\mathrm{x}} \mathrm{S}_{\mathrm{x}}[21,22]$ acquired broad attention due to the possibility of $\mathrm{ZnO}$ band structure modifications, making the alloys useful for solar applications.
The following parameters, typical for $\mathrm{ZnO}$, were used for the calculations: the effective masses and heavy holes were considered as the same for all layers, but different for the $z$-direction and in the transverse $z$ direction, hereinafter denoted by the superscript $\|$ (the structure growth $z$-axis was chosen along the $c$-axis of the crystal), namely: $m_{e z}=0.23 m_{0}$ and $m_{e \|}=0.21 m_{0}$ for electrons, and $m_{h h z}=0.74 m_{0}$ and $m_{h \|}=0.54 m_{0}$ for holes, where $m_{0}$ is the mass of the free electron; the dielectric permittivity $\varepsilon=7.4[23,24]$. The quantum barrier height for electrons in the conduction band and the well depth for heavy holes in the valence band are not precisely known. We supposed that heavy holes are well confined in the deep quantum well with the depth $V_{h}=1 \mathrm{eV}$ (which corresponds to the real band structure $[8,22])$. The electron barrier height was a parameter for these calculations, although according to the the band edge calculations [22] it is about $V_{e}=0.1 \mathrm{eV}$ for the $\mathrm{ZnO} / \mathrm{ZnO}_{1-\mathrm{x}} \mathrm{Te}_{\mathrm{x}} / \mathrm{ZnO}$ structure with $\mathrm{Te}$ content of about $x=0.8$.

The variational calculations of the lowest $1 \mathrm{~S}$ Wannier exciton state were carried out using two trial functions $\Psi_{c}\left(\vec{r}_{e}, \vec{r}_{h}\right)$ (2) and $\Psi_{n c}\left(\vec{r}_{e}, \vec{r}_{h}\right)$ (4). The first one, $\Psi_{c}\left(\vec{r}_{e}, \vec{r}_{h}\right)$, doesn't allow the mentioned shift of carriers towards the structure interfaces and fixes the central position of hole in the well, and is traditional for calculations concerning similar systems [17, 18]. The second trial function, $\Psi_{n c}\left(\vec{r}_{e}, \vec{r}_{h}\right)$, the proposed one, is suitable for searching for a possible solution with the shifted hole. If the energy obtained for the state with the "non-central" hole position turns out to be less than obtained for the "central" hole position, this shifted state can be realized in the structure as an energetically favorable.

\section{Results and discussion}

In Fig. 2, the exciton binding energy calculated using both trial functions $\Psi_{c}\left(\vec{r}_{e}, \vec{r}_{h}\right)$ (2) and $\Psi_{n c}\left(\vec{r}_{e}, \vec{r}_{h}\right)$ (4) in dependence on the internal layer width is shown (the internal layer width means the barrier width for electrons and the well width for holes). In this structure, the exciton binding energy should be calculated as the difference between the exciton transition energy and the transition energy for an uncoupled electron-hole pair. It means that in this case the energy of the lowest one-particle hole state in the quantum well (in the absence of Coulomb coupling the electron is free, and its energy is equal to zero). As the quantum well becomes wider, the distance between the carriers increases, and the Coulomb attraction becomes weaker, and, as a result, the binding energy absolute value rises. As can be seen, it takes place for the binding energies calculated using the both trial functions till the width of about $15 \mathrm{~nm}$. At further increase of the well width, the binding energy calculated using the "central" 


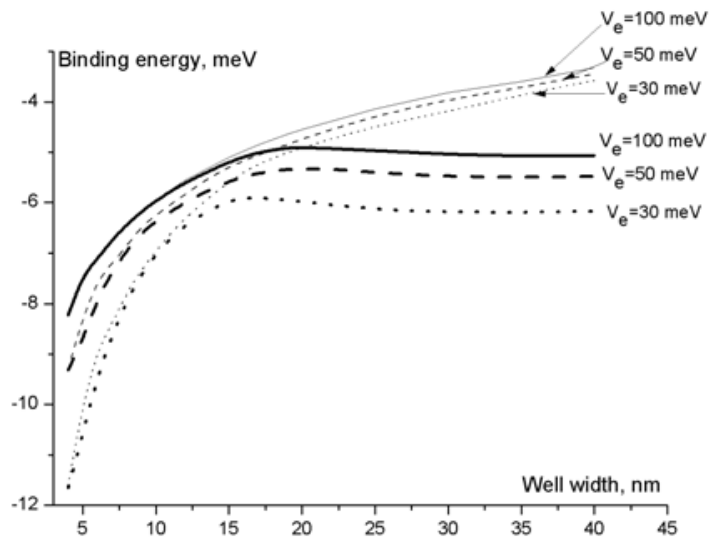

Fig. 2. The exciton binding energy versus the well width for the hole well depth equal to $V_{h}=1 \mathrm{eV}$ and several values of the barrier height for electron. The results calculated using the trial functions $\Psi_{c}\left(\vec{r}_{e}, \vec{r}_{h}\right)$ and $\Psi_{n c}\left(\vec{r}_{e}, \vec{r}_{h}\right)$ are shown by thin and thick curves, correspondingly.

function $\Psi_{c}\left(\vec{r}_{e}, \vec{r}_{h}\right)$ continues to reduce in its absolute value (thin curves in Fig. 2). It is related, again, with the increase of the distance between the particles. Though, for these wide wells, the wave function $\Psi_{c}\left(\vec{r}_{e}, \vec{r}_{h}\right)$ can be hardly used - the width of the well exceeds by several times the Bohr radius of bulk exciton (approximately $2 \ldots 2.5 \mathrm{~nm}$ ), and the distance between the hole levels in the well becomes less than the exciton binding energy. Contrary to these results, the binding energy calculated using the "non-central" trial function $\Psi_{n c}\left(\vec{r}_{e}, \vec{r}_{h}\right)$ for wide wells doesn't change essentially but even slightly reduces as the well width rises (thick curves in Fig. 2). It takes place because the hole moves to the interfaces reducing the distance to electron, so Coulomb interaction remains strong. Of course, the one-particle function for hole is also used as a multiplier in the function $\Psi_{n c}\left(\vec{r}_{e}, \vec{r}_{h}\right)$, but as calculations show in fact the hole is localized in a thin (about the Bohr radius wide) region near the interfaces. For the presented results, the variational parameter $\gamma$ determining the shift of the hole position is equal $\gamma L / 2=0.833$ for the well width $L=10 \mathrm{~nm}$ and $\gamma L / 2=2.6$ for the well width $L=20 \mathrm{~nm}$. In order to demonstrate the spatial positions of the carriers, the components of the exciton wave functions describing the electron and hole positions in the well are shown in Fig. 3. The wider well is, the more the functions differ from each other. The results obtained for the function $\Psi_{n c}\left(\vec{r}_{e}, \vec{r}_{h}\right)$ (4), which allows describing the shift of the hole, show that both the electron and the hole in a wide well tend to approach closer to the interfaces of the structure, increasing Coulomb binding. For narrow wells, the results obtained with both functions are very close. In order to illustrate the trial functions in a slightly different way, in Fig. 4 the probability density distributions of finding the electron at $z_{e}$ and hole at $z_{h}$ $p_{(c) n c}\left(z_{e}, z_{h}\right)=2 \pi \int \Psi_{(c) n c}\left(\vec{r}_{e}, \vec{r}_{h}\right) \rho d \rho$ is calculated using both these trial functions are shown. Although the oneparticle functions $f_{e}\left(z_{e}\right)$ and $f_{h, c}(n c)\left(z_{h}\right)$ are symmetric in respect to $z_{e}=0$ and $z_{h}=0$ axes, respectively, but the probability density is symmetric in respect to the $z_{e}=-z_{h} z_{e}=-z_{h}$ axis. It is caused by the use of the $3 D$

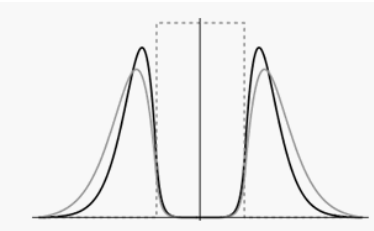

a)

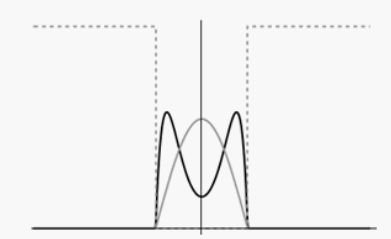

d)

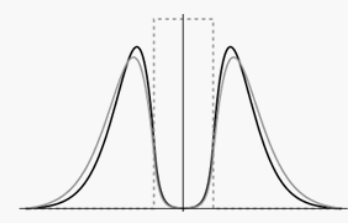

b)

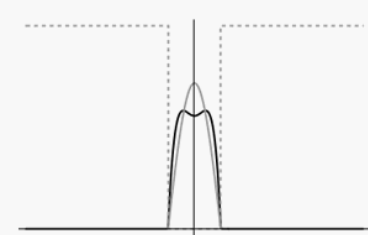

e)

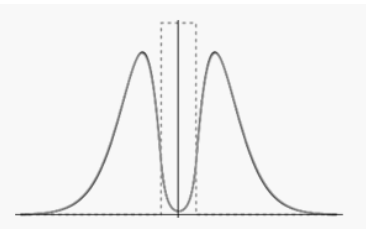

c)

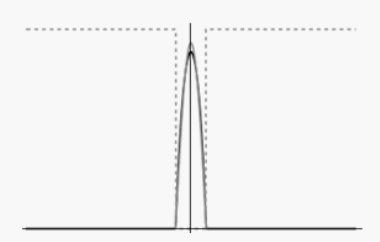

f)

Fig. 3. The one-particle components of the total exciton function describing the electron and hole positions in the structure. The upper row $(a-c)$ : the electron one-particle function $f_{e}\left(z_{e}\right)$ obtained numerically for the proposed "non-central" trial function $\Psi_{n c}\left(\vec{r}_{e}, \vec{r}_{h}\right)$ (black) and for the "central" function $\Psi_{c}\left(\vec{r}_{e}, \vec{r}_{h}\right)$ (gray). The lower row $(d-f)$ : the hole one-particle functions $f_{h, n c}\left(z_{h}\right)$ (black) and $f_{h, c}\left(z_{h}\right)$ (gray). The calculations were carried out for the 30-nm $(a, d), 17-\mathrm{nm}(b, e)$ and 10-nm (c,f) width wells, respectively, and for $V_{e}=100 \mathrm{meV}, V_{h}=1 \mathrm{eV}$. The dashed lines depict schematically the potential barrier for electrons and the potential well for holes. 
trial function (6) reducing exponentially as $\left|z_{e}-z_{h}\right|$ rises. It can be seen in Figs. 3, 4 that in the state described by the proposed trial function $\Psi_{n c}\left(\vec{r}_{e}, \vec{r}_{h}\right)$ the carriers are localized in a narrow (of about bulk Bohr radius) area near the interface that allows suggesting that this trial function can be used for wide quantum wells, where the use of traditional function $\Psi_{c}\left(\vec{r}_{e}, \vec{r}_{h}\right)$ is definitely not justified.

Although the binding energy is an important parameter of the exciton state, it is the exciton transition energy that can be observed experimentally. As one can see in Fig. 5, at the beginning the exciton transition energy decreases as the well width rises because of the lowering the one-particle hole level. In very wide wells this one-particle level is so low that its effect is vanishing, and the transition energy calculated by the "central" function $\Psi_{c}\left(\vec{r}_{e}, \vec{r}_{h}\right)$ rises because of reduction

of Coulomb interaction (thin curves in Fig. 5). The transition energy obtained using the "non-central" function $\Psi_{n c}\left(\vec{r}_{e}, \vec{r}_{h}\right)$ slowly decreases as the well width rises because of the hole position shift and strengthening Coulomb interaction explained above. In this region, the energy obtained with the "shifted" trial function is less than the energy obtained with the "central" one, which justify the use of the proposed "shifted" function (4).
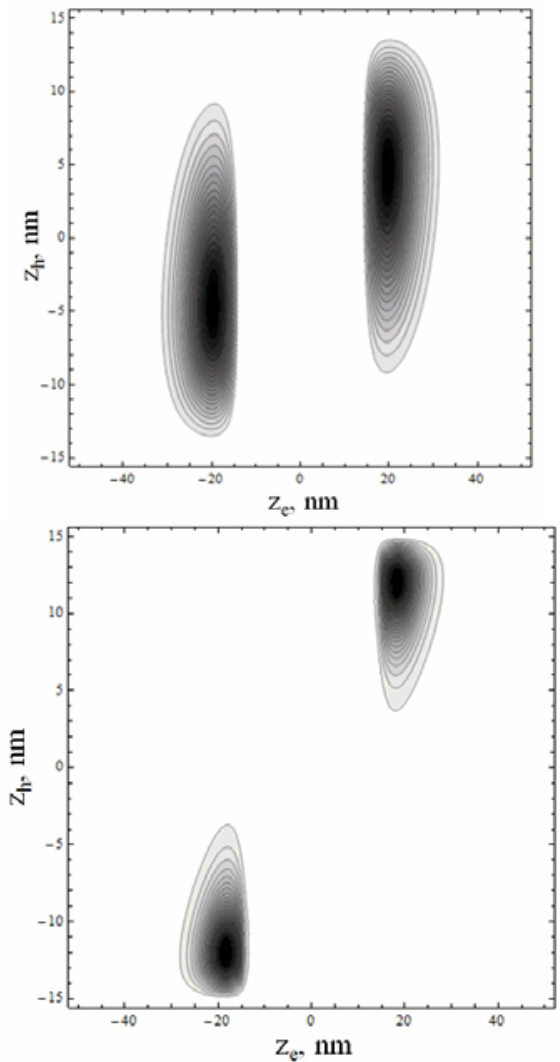

Fig. 4. The probability density distributions of finding the electron at $z_{e}$ and hole at $z_{h}$ for the $30 \mathrm{~nm}$ well and the band offsets $V_{e}=100 \mathrm{meV}$ and $V_{h}=1 \mathrm{eV}$ calculated using the trial functions $\Psi_{c}\left(\vec{r}_{e}, \vec{r}_{h}\right)(a)$ and $\Psi_{n c}\left(\vec{r}_{e}, \vec{r}_{h}\right)(b)$.
In this system, the exciton energy essentially depends on the band discontinuities at interfaces, which determines to what extent the carriers are separated. In other words, the higher the barrier for the electron is, the stronger this separation and the less the exciton binding energy are. Lowering the barrier for the electron at the fixed well depth for the hole leads to the deeper penetration of the electron wave function into the barrier layer, where the hole is confined, which means the stronger overlap of the wave functions of the carriers and their stronger coupling. It can be seen in Fig. 6, where the dependence of the binding energy on the electron barrier height in the conduction band is presented for the fixed well depth for the hole for a few well widths. At some point, the barrier height becomes so small that the electron moves into the barrier layer, and the spatially indirect exciton turns into the direct exciton. This change of the spatial state of exciton manifests itself into a strong increase of the absolute value of the binding energy (Fig. 6).

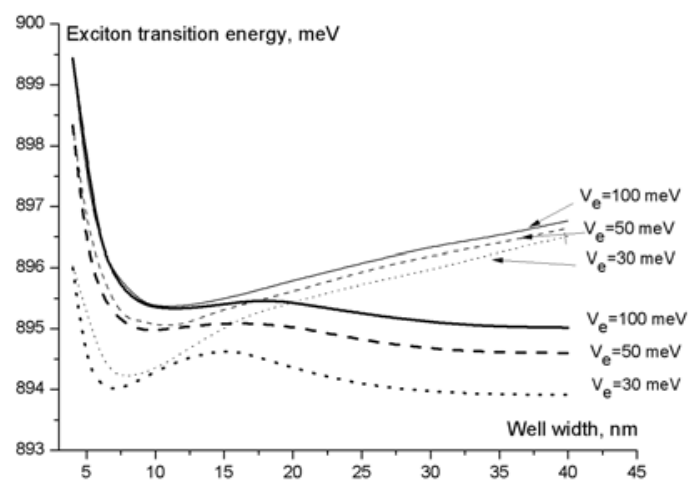

Fig. 5. The exciton transition energy versus the well width for the hole well depth equal to $V_{e}=0.1 \mathrm{eV}$ and a few values of the barrier height for the electron in the conduction band. The results calculated using the trial functions $\Psi_{c}\left(\vec{r}_{e}, \vec{r}_{h}\right)$ and $\Psi_{n c}\left(\vec{r}_{e}, \vec{r}_{h}\right)$ are shown by thin and thick curves, correspondingly. The band gap of the internal $\mathrm{ZnO}_{1-\mathrm{x}} \mathrm{Te}_{\mathrm{x}}$ level was taken here as equal to $1 \mathrm{eV}$, which corresponds to the $\mathrm{ZnO}_{0.2} \mathrm{Te}_{0.8}$ layer [22].

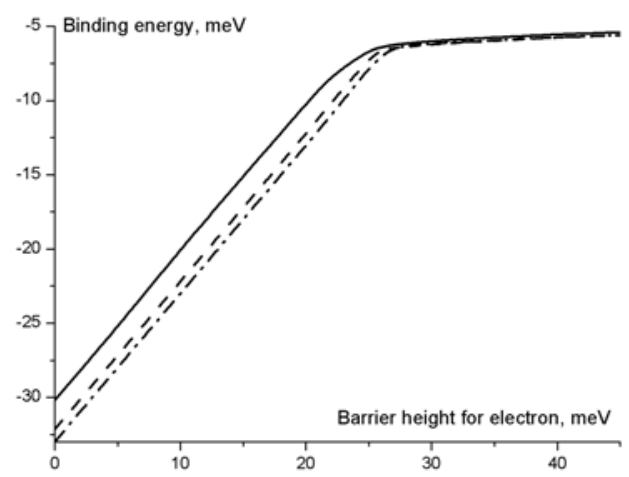

Fig. 6. The exciton binding energy versus the barrier height for electrons in the conduction band calculated with the proposed trial function $\Psi_{n c}\left(\vec{r}_{e}, \vec{r}_{h}\right)$ for $V_{h}=1 \mathrm{eV}$ for the 20-nm (solid curve), 30-nm (dash) and 40-nm (dash dot) width wells. 


\section{Conclusions}

For a type-II single quantum well, a trial variational exciton wave function allowing calculation of the binding energy value for wide wells was proposed. A single quantum well with hole confined in the potential well of the internal layer of the structure and electrons localized in the external layers was considered. The proposed wave function takes into account that due to strong Coulomb interaction the hole can approach to the interfaces, reducing the distance to electron, and this spatial state can be more energetically favorable than the state with the most probable position of the hole in the center of the well. For $\mathrm{ZnO}$ based quantum wells, the $1 \mathrm{~S}$ Wannier exciton state transition energy and the binding energy were calculated variationally using two trial functions: a traditional function with hole localized in the center of the well and the proposed trial function with "shifted" hole. It was shown that in the shifted state hole is localized near the interfaces at the distance of about the Bohr radius for bulk exciton from the electron, which can justify the use of this trial functions for wide quantum wells were the traditional trial function cannot be used.

\section{Acknowledgements}

The author expresses her gratitude to Prof. V.I. Sugakov and Dr. I.Yu. Goliney for formulation of the problem and the useful discussions.

\section{References}

1. A. Reznitsky, S. Permogorov, S. Verbin, A. Naumov, Yu. Korostelin, V. Novozhilov, S. Prokof'ev, Localization of excitons and Anderson transition in $\mathrm{ZnSe}_{1-\mathrm{x}} \mathrm{Te}_{\mathrm{x}}$ solid solutions // Solid State Communs. 52, p. 13-16 (1984).

2. Chin-Hau Chia, Wen-Chung Fan, Yan-Chen Lin, Wu-Ching Chou, Radiative recombination of indirect exciton in type-II $\mathrm{ZnSeTe} / \mathrm{ZnSe}$ multiple quantum wells // J. Lumin. 131, p. 956-959 (2011).

3. U.E.H. Laheld, F.B. Pederson, P.C. Hemmer, Excitons in type-II quantum dots: Finite offsets // Phys. Rev. B, 52, p. 2697-2703 (1995).

4. G.G. Zegrya, A.D. Andreev, Mechanism of suppression of Auger recombination processes in type-II heterostructures // Appl. Phys. Lett. 67, p. 2681-2683 (1995).

5. A.I. Yakimov, A.V. Dvurechenskii, A.I. Nikiforov, V.V. Ivanov, A.G. Milekhin, A.O. Govorov, S. Schulze, D.R.T. Zahn, Stark effect in type-II Ge/Si quantum dots // Phys. Rev. B, 67, p. 125318 (2003).

6. R.J. Warburton, C. Schulhauser, D. Haft, C. Schaflein, K. Karrai, J.M. Garcia, W. Schoenfeld, P.M. Petroff, Giant permanent dipole moments of excitons in semiconductor nanostructures // Phys. Rev. B, 65, p. 113303 (2002).
7. I. Vurgaftman, J.R. Meyer, N. Tansu, and L.J. Mawst, (In)GaAsN-GaAsSb type-II "W" quantum-well lasers for emission at $\lambda=1.55 \mu \mathrm{m} / /$ Appl. Phys. Lett. 83, p. 2742-2744 (2003).

8. J. Schrier, D.O. Demchenko, L.W. Wang, and A.P. Alivisatos, Optical properties of $\mathrm{ZnO} / \mathrm{ZnS}$ and $\mathrm{ZnO} / \mathrm{ZnTe}$ heterostructures for photovoltaic applications // Nano Lett. 7, p. 2377-2382 (2007).

9. A.R. Giehl, M. Gumbel, C. Schwender, N. Herhammer, and H. Fouckhardt, Waveguidebased type-II heterostructure photodiode on InAs substrate with broad wavelength range photoresponse // IEEE Photonics Technol. Lett. 16, p. 1358-1360 (2004).

10. R. Sidhu, N. Duan, J. Campbell and A. Holmes, A long-wavelength photodiode on InP using latticematched GaInAs-GaAsSb type-II quantum wells // IEEE Photon. Technol. Lett. 17, p. 2715-2717 (2005).

11. H.S. Kim, O.O. Cellek, Zhi-Yuan Lin, Zhao-Yu He, Xin-Hao Zhao, Shi Liu, H. Li, and Y.-H. Zhanga, Long-wave infrared $\mathrm{nBn}$ photodetectors based on InAs/InAsSb type-II superlattices // Appl. Phys. Lett. 101, p. 161114 (2012).

12. M.A. Petruska, A. Malko, P.M. Voyles, and V.I. Klimov, High-performance, quantum dot nanocomposites for nonlinear optical and optical gain applications // Adv. Mater. 7-8, p. 610-613 (2003).

13. V.S. Bagaev, V.S. Krivobok, S.N. Nikolaev, A.V. Novikov, E.E. Onishchenko, M.L. Skorikov, Observation of the electron-hole liquid in $\mathrm{Si}_{1-\mathrm{x}} \mathrm{Ge}_{\mathrm{x}} / \mathrm{Si}$ quantum wells by steady state and time resolved photoluminescence measurements // Phys. Rev. B, 82, p. 115313 (2010).

14. T.M. Burbaev, M.N. Gordeev, D.N. Lobanov, A.V. Novikov, M.M. Rzaev, N. N. Sibel'din, M.L. Skorikov, V.A. Tsvetkov, and D.V. Shepel', Electron-hole liquid and excitonic molecules in quasi-two-dimensional $\mathrm{SiGe}$ layers of $\mathrm{Si} / \mathrm{SiGe} / \mathrm{Si}$ heterostructures // JETP Lett. 92, p. 305-309 (2010).

15. T. Baier, U. Mantz, K. Thonke, and R. Sauer, F. Schaffier and H.-J. Herzog, Type-II band alignment in $\mathrm{Si} / \mathrm{Si}_{1-\mathrm{x}} \mathrm{Ge}_{\mathrm{x}}$ quantum wells from photoluminescence line shifts due to optically induced band-bending effects: Experiment and theory // Phys. Rev. B, 50, p. 15191-15196 (1994).

16. M. Matsuura and Y. Shinozuka, Excitons in type-II quantum-well systems: Binding of the spatially separated electron and hole // Phys. Rev. B, 38, p. 9830-9837(1988).

17. A. Bellabchara, P. Lefebvre, P. Christol, and H. Mathieu, Improved modeling of excitons in type-II semiconductor heterostructures by use of a three-dimensional variational function // Phys. Rev. B, 50, p. 11840-11844 (1994).

18. A. Chaves, J. Costa e Silva, J.A.K. Freire and G.A. Farias, Excitonic properties of type-I and 
type-II Si $/ \mathrm{Si}_{1-\mathrm{x}} \mathrm{Ge}_{\mathrm{x}}$ quantum wells // J. Appl. Phys. 101, p. 113703 (2007).

19. H.Y. Chao, J.H. Cheng, J.Y. Lu, Y.H. Chang, C.L. Cheng, Y.F. Chen, Growth and characterization of type-II $\mathrm{ZnO} / \mathrm{ZnTe}$ core-shell nanowire arrays for solar cell applications // Superlattices and Microstructures, 47, p. 160-164 (2010).

20. H.L. Pan, T. Yang, B. Yao, R. Deng, R.Y. Sui, L.L. Gao, and D.Z. Shen, Characterization and properties of $\mathrm{ZnO}_{1-\mathrm{x}} \mathrm{S}_{\mathrm{x}}$ alloy films fabricated by radio-frequency magnetron sputtering // Appl. Surf. Sci. 256, p. 4621-4625 (2010).

21. M. Jaquez, K.M. Yu, M. Ting, M. Hettick, J.F. Sanchez-Royo, M. Wełna, A. Javey,
O.D. Dubon, and W. Walukiewicz, Growth and characterization of $\mathrm{ZnO}_{1-\mathrm{x}} \mathrm{S}_{\mathrm{x}}$ highly mismatched alloys over the entire composition // J. Appl. Phys. 118, p. 215702 (2015).

22. M. Ting, R. dos Reis, M. Jaquez, O.D. Dubon, S.S. Mao, K.M. Yu, and W. Walukiewicz, Electronic band structure of $\mathrm{ZnO}$-rich highly mismatched $\mathrm{ZnO}_{1-\mathrm{x}} \mathrm{Te}_{\mathrm{x}}$ alloys // Appl. Phys. Lett. 106, p. 092101 (2015).

23. W.R.L. Lambrecht, A.V. Rodina, S. Limpijumnong, B. Segall, and B.K. Meyer, Valence-band ordering and magneto-optic exciton fine structure in $\mathrm{ZnO} / /$ Phys. Rev. B, 65, p. 075207 (2002).

24. H. Yoshikawa, S. Adachi, Optical constants of $\mathrm{ZnO}$ // Jpn. J. Appl. Phys. 36, p. 6237 (1997). 